\title{
Differences in cerebral cortical anatomy of left- and right-handers
}

\section{Tulio Guadalupe ${ }^{1,2}$, Roel M. Willems ${ }^{3,4}$, Marcel P. Zwiers ${ }^{3}$, Alejandro Arias Vasquez ${ }^{5,6,7}$, Martine Hoogman ${ }^{1,5}$, Peter Hagoort ${ }^{3,4}$, Guillen Fernandez ${ }^{3,7}$, Jan Buitelaar ${ }^{6,7}$, Barbara Franke ${ }^{5,6,7}$, Simon E. Fisher ${ }^{1,8}$ and Clyde Francks ${ }^{1,8 *}$}

1 Language and Genetics Department, Max Planck Institute for Psycholinguistics, Nijmegen, Netherlands

2 International Max Planck Research School for Language Sciences, Nijmegen, Netherlands

${ }^{3}$ Centre for Cognitive Neuroimaging, Donders Institute for Brain, Cognition and Behaviour, Radboud University Nijmegen, Nijmegen, Netherlands

${ }^{4}$ Neurobiology of Language Department, Max Planck Institute for Psycholinguistics, Nijmegen, Netherlands

${ }^{5}$ Department of Human Genetics, Radboud University Medical Center, Nijmegen, Netherlands

${ }^{6}$ Department of Psychiatry, Donders Institute for Brain, Cognition and Behaviour, Radboud University Medical Center, Nijmegen, Netherlands

7 Department of Cognitive Neuroscience, Donders Institute for Brain, Cognition and Behaviour, Radboud University Medical Center, Nijmegen, Netherlands

${ }^{8}$ Donders Institute for Brain, Cognition and Behavior, Radboud University Nijmegen, Nijmegen, Netherlands

\section{Edited by:}

Sebastian Ocklenburg, University of Bergen, Norway

Reviewed by:

René Westerhausen, University of Bergen, Norway

Isabelle Silvia Haberling, University of Auckland, New Zealand

*Correspondence:

Clyde Francks, Language and Genetics Department, Max Planck Institute for Psycholinguistics, Wundtlaan 1, Nijmegen 6525 XD, Netherlands

e-mail: clyde.francks@mpi.nl
The left and right sides of the human brain are specialized for different kinds of information processing, and much of our cognition is lateralized to an extent toward one side or the other. Handedness is a reflection of nervous system lateralization. Roughly ten percent of people are mixed- or left-handed, and they show an elevated rate of reductions or reversals of some cerebral functional asymmetries compared to right-handers. Brain anatomical correlates of left-handedness have also been suggested. However, the relationships of left-handedness to brain structure and function remain far from clear. We carried out a comprehensive analysis of cortical surface area differences between 106 left-handed subjects and 1960 right-handed subjects, measured using an automated method of regional parcellation (FreeSurfer, Destrieux atlas). This is the largest study sample that has so far been used in relation to this issue. No individual cortical region showed an association with left-handedness that survived statistical correction for multiple testing, although there was a nominally significant association with the surface area of a previously implicated region: the left precentral sulcus. Identifying brain structural correlates of handedness may prove useful for genetic studies of cerebral asymmetries, as well as providing new avenues for the study of relations between handedness, cerebral lateralization and cognition.

Keywords: MRI, handedness, cortical surface area, brain asymmetry, FreeSurfer

\section{INTRODUCTION}

Handedness is perhaps the most overt reflection of lateralization of the central nervous system in humans. Humans show a strong and population-level bias toward using one hand rather than the other for manual activities, which is unusual among mammals (Vallortigara et al., 2011). Roughly 90\% of humans are right-handed, while even other primates (e.g., chimpanzees and macaques) do not show such a strong degree of populationlevel handedness (Lonsdorf and Hopkins, 2005; Meunier et al., 2013). This motor asymmetry is observable at least as early during human development as 15 weeks of gestation, and is preceded by asymmetries of arm movements even earlier (Hepper, 2013). In addition the tendency toward right handedness has apparently been present throughout human history, and across cultures and continents (Coren and Porac, 1977; Hardyck and Petrinovich, 1977; McManus, 1991, 2009; Faurie and Raymond, 2004).

Due in part perhaps to its minority status and past cultural stigmatization, left-handedness has often been studied in the context of pathology, for example in relation to Alzheimer's disease (de Leon et al., 1986), substance use (London, 1989), and autoimmune disorders (Geschwind and Behan, 1982). Handedness has also been investigated in relationship to lateralized cognitive functions, such as visuospatial processing (Gordon and Kravetz, 1991), face recognition (Luh et al., 1994; Willems et al., 2010; Bukowski et al., 2013) and prominently, language (Tzourio et al., 1998; Knecht et al., 2000b). Knecht and colleagues found an increased incidence of bilateral and right hemisphere language lateralization among left-handers, compared to righthanders, although the majority of left/mixed handers still showed left-hemisphere language dominance (Knecht et al., 2000a,b). This suggests that developmental mechanisms affecting cerebral language dominance overlap to an extent with those influencing hand motor control. However, it remains poorly understood how these different domains of functional lateralization are related to each other (Badzakova-Trajkov et al., 2010).

Several early attempts to understand human handedness attributed right-handedness to socio-cultural, anatomical, as well as genetic factors (for a review see Hardyck and Petrinovich, 1977 or Corballis et al., 2012 for a more recent one). However, the developmental basis of human brain lateralization remains 
almost wholly unknown, and likewise the causes of its variation are hardly understood (Willems et al., 2014). One robust observation is that males show a slightly higher proportion of lefthandedness than females (Halpern et al., 1998; Peters et al., 2006; Sommer et al., 2008). Recent twin studies, based on thousands of families, have indicated that $21-24 \%$ of the liability to lefthandedness can be explained by additive genetic effects (Medland et al., 2009; Vuoksimaa et al., 2009). This indicates that genetic variation plays a role in causing variation in handedness. In contrast to original models of handedness as a monogenic trait (Annett, 1985; McManus, 1985), recent evidence from genomewide association studies strongly suggest more complex models (Medland, 2009; McManus et al., 2013; Armour et al., 2014). So far, studies aimed at discovering the specific genetic loci involved have yielded tentative associations with the genes AR, APOE, COMT, PCSK6, LRRTM1 (Medland et al., 2005; Francks et al., 2007; Savitz et al., 2007; Bloss et al., 2010; Scerri et al., 2011; Brandler et al., 2013). Although originally discovered in populations affected by dyslexia, PCSK6 has also shown association with degree of handedness in a healthy sample of unrelated adults (Arning et al., 2013). It is not yet known how these genes may influence asymmetrical development of the brain (see Ocklenburg et al., 2013).

Identifying brain anatomical correlates of left-handedness may provide potential endophenotypes for further genetic association studies (Ocklenburg et al., 2013; Willems et al., 2014). Finding anatomical correlates of left-handedness may also inform on the relations between handedness and lateralized cognitive functions, and more broadly on brain structure-function relationships (Ocklenburg et al., 2013; Willems et al., 2014). Amunts et al. (1996) found deeper left precentral sulci in right-handers than left-handers using manual segmentations of magnetic resonance (MR) images. Consistent with this, Foundas et al. (1998) examined left-right asymmetries of the precentral gyrus in a sample of 15 left- and 15 right handers based on manual segmentations of their MR images, and found leftward asymmetries in righthanders, but no consistent asymmetry in left-handers (also see Kloppel et al., 2007 and Willems and Hagoort, 2009, for corroborating findings using functional MR imaging). More recently, gray matter volume in the central sulcus was shown to relate to hand motor skill, but to different extents depending on handedness (Herve et al., 2005). In addition, asymmetry of the planum temporale (PT), the posterior portion of the superior surface of the temporal lobe, has been reported to associate with hand preference (Steinmetz et al., 1991; Foundas et al., 1995; Herve et al., 2006). However, results regarding the PT have not been consistent throughout the literature (Witelson and Kigar, 1992; Good et al., 2001). Similarly, an association between handedness and cerebral torque, another structural brain asymmetry, has also been assessed with inconclusive results (Narr et al., 2007). More recently, Powell et al. (2012) in a study of 40 left-handers and 42 right-handers found differences in sulcal shape of the pars orbitalis (PO) and pars triangularis (PTr), as well as differences of volumetric asymmetry within the PO. To our knowledge, Good et al. (2001) has studied the largest sample to have been used in examining brain morphological differences related to handedness. Using a voxel-based morphometry analysis with a total sample of 465 subjects (67 lefthanders) they did not find structural correlates of handedness in the brain. This suggests that any such correlates are subtle and will require larger samples and/or other ways to quantify brain structure, in order to detect them unambiguously.

The goal of the present study was to identify cerebral cortical differences between left and right-handers, by analyzing the largest sample used so far for this purpose (106 left-handed subjects and 1960 right-handed subjects), and using recently developed methodology for the automated segmentation and quantification of regional gray matter (Fischl et al., 2004). We analyzed the data in three stages. First we examined total cortical surface area in relation to handedness. Then, we tested a set of candidate cortical regions for associations with handedness, based on the previous studies mentioned above. Finally, we carried out a screen over all remaining cortical regions.

\section{METHODS \\ STUDY DATASET}

The Brain Imaging Genetics (BIG) study was initiated in 2007 and comprises healthy volunteer subjects, including many university students, who participate in diverse imaging studies at the Donders Centre for Cognitive Neuroimaging (DCCN), Nijmegen, The Netherlands (Franke et al., 2010). At the time of this study the BIG subject-pool consisted of 2337 self-reported healthy individuals (1248 females) who had undergone anatomical (T1-weighted) MRI scans, usually as part of their involvement in diverse smaller-scale studies at the DCCN, and who had given their consent to participate in BIG. Their median age was 23 years. A subset of 235 subjects had undergone a brain MRI scan twice, with at least 1 day separation between scans. Fifty percent of the 235 re-scans took place within 181 days of the first, with the mean elapsed time being 320 days $(S D=360)$. At the time of the first scan, the median age of this group was 23 years.

Handedness of the participants was assessed by an item in their enrolment form. This consisted of subjects selecting the appropriate label, either "left-handed/right-handed" (in Dutch). We discuss the validity of this method of assessing handedness further below. Only those subjects who clearly indicated one or the other state were included in our analysis. This resulted in a sample of 1960 right-handed subjects and 106 left-handed subjects, with a median age of 22 years and a standard deviation of 11 years. The proportion of left-handers was substantially lower than in the general population; this was due to left- handedness being used as an exclusion criterion for some of the imaging studies that were pooled into the overall BIG dataset.

\section{IMAGE ACOUISITION}

MRI data in BIG were acquired with either a 1.5 Tesla Siemens Sonata or Avanto scanner or a 3 Tesla Siemens Trio or TimTrio scanner (Siemens Medical Systems, Erlangen, Germany). Given that images were acquired during several smaller scale studies, the parameters used were slight variations of a standard T1-weighted three-dimensional magnetization prepared rapid gradient echo sequence (MPRAGE; $1.0 \times 1.0 \times 1.0 \mathrm{~mm}$ voxel size). The most common variations in the TR/TI/TE/sagittal-slices parameters were the following: 2300/1100/3.03/192, 2730/1000/2.95/176, 
2250/850/2.95/176, 2250/850/3.93/176, 2250/850/3.68/176, 2300/1100/3.03/192, 2300/1100/2.92/192, 2300/1100/2.96/192, 2300/1100/2.99/192, 1940/1100/3.93/176 and 1960/1100/4.58/ 176. There was also variation in the number of headcoils used across BIG scans, however, no systematic differences were observed in their use between left- and right-handed subjects. The following arrays were employed (and their frequencies) in the right-handed population: 32-channel (24\%), 12-channel (4\%), 8-channel (38\%), arrays and single headcoil (33\%). In the left-handed population, this distribution was 32-channel (27\%), 12-channel $(0 \%)$, 8-channel $(33 \%)$, arrays and single headcoil $(40 \%)$.

\section{IMAGE PROCESSING}

Automated parcellation of cerebral cortical regions from T1weighted images was done in FreeSurfer v5.1 (Fischl et al., 2004) according to the Destrieux atlas (Destrieux et al., 2010) within the "-recon-all" processing pipeline, and using default parameters. Measures of surface area (in $\mathrm{mm}^{2}$ ) were produced for the total cortical surface and for each of 74 cortical parcellations, in each hemisphere. Outlier values (more extreme than $3.5 \mathrm{SD}$ from the mean) were excluded for each measure. The scan-rescan correlation of each measure was then calculated in the sample of 235 subjects who had undergone two MRI scans, after correcting for the potential covariate effects of age, sex, total cortical surface area and scanner field strength (IBM SPSS v.20).

Out of the 74 covariate-corrected bilateral cortical measures, 23 were excluded from subsequent analyses, due to low scanrescan correlation in either left, right or both structures (Pearson's $r<0.7$; i.e., corresponding to shared proportion of variance between scan and re-scan measures of $<0.49$ ). Regional measures of cortical thickness were also generated. There is evidence that cortical surface and thickness have independent sources of variation (Panizzon et al., 2009). However, we discarded the thickness measures because the majority $(81 \%)$ showed scan-rescan correlations below 0.7 .

\section{CORTICAL CORRELATES OF HANDEDNESS}

We tested for associations between handedness and cortical surface areas using repeated-measures ANOVA, implemented in SPSS (IBM SPSS v.20). Hemisphere (left vs. right) was factored as a within-subjects variable and handedness group as a between-subjects variable in a full factorial design. This allowed the detection of bilateral associations of handedness with cortical surface areas, as well as asymmetrical associations (by means of the interaction between handedness and hemisphere). We first tested the total hemispheric surface areas, and then we tested the regional surface areas. In addition, the following covariates were entered into the analyses: sex, age, scanner field strength, and total (i.e., left plus right) hemispheric surface area (the latter only for the analyses of regional surfaces).

We tested candidate cortical regions motivated by previous findings in the literature (specifically by the studies reviewed in the introduction). We separated these candidate regions into three domains; language, motor control and visual processing. Language-related candidate regions were the inferior frontal gyrus and superior temporal gyrus. These corresponded most closely to the following parcellations within the Destrieux atlas, that had also showed a robust scan-rescan correlation: Opercular part of the inferior frontal gyrus, triangular part of the inferior frontal gyrus, anterior transverse temporal gyrus (of Heschl), lateral aspect of the superior temporal gyrus, and PT. The motor control candidate regions were the superior and inferior parts of the precentral sulcus (as defined in the Destrieux atlas). The visual-related candidate regions comprised inferior and ventral areas of the temporal lobe. In the Destrieux atlas these corresponded most closely to the following regions: inferior temporal gyrus, lateral occipito-temporal gyrus (fusiform gyrus) and lingual part of the medial occipito-temporal gyrus. We applied Bonferroni corrections for the comparisons done within each of these domains.

After the analysis of candidate regions, we then tested all of the remaining cortical regions for differences between left- and right-handers, again using Bonferroni adjustment to correct for multiple testing.

\section{POWER ANALYSIS}

We used G* Power v3.1.9 (Faul et al., 2009) to estimate the necessary effect sizes to be detected given our study design. We considered our sample size, a required power $(1-\beta)$ of $80 \%$, a correlation between bilateral volumes of $r \sim 0.8$, and an $\alpha$ level corrected for multiple testing. This resulted in estimates of partial $\eta^{2} \sim 0.07\left[F_{(1,2055)} \sim 5.7\right]$ for analyses within each of the candidate domains, and a partial $\eta^{2} \sim 0.09\left[F_{(1,2055)} \sim 10\right]$ for the analysis of the remaining cortical surfaces. In other words we had $80 \%$ power to detect an association explaining $9 \%$ of the residual variance in a regional cortical surface area after having removed the effects of covariates and after considering the multiple comparisons, for the screening analysis of non-candidate regions.

\section{RESULTS}

The proportion of left-handers in our sample differed significantly between males and females. Of the 942 males, 59 were left-handed (6.3\%), and of the 1077 females, 47 were left-handed $(4.4 \%) ; \chi_{(1)}^{2}=4.56, p=0.02$, phi $=0.047$.

Handedness did not show a significant association with bilateral hemispheric surface area, nor with overall hemispheric surface asymmetry (see Tables 1, 2). None of the candidate regions, related to either language, visual processing, or motor control showed significant evidence for association with handedness after correction for multiple testing within each of these domains (see Table 3). The only regions showing main effects of handedness with $p<0.05$ before correction for multiple testing were the superior precentral sulcus and the inferior temporal gyrus. Means (and SDs) for these regions, by hemisphere and handedness group, are shown in Table 4.

Tables 5, 6 show results for the remaining (non-candidate) regional surface areas that reached nominal significance (i.e., uncorrected $p<0.05$ ) for an association with handedness, either as a main effect on bilateral surface or as an interaction with hemisphere. None of these associations survived correction for multiple testing. The results for all cortical regions and covariates, regardless of nominal significance, can be found in 
Table 1 | Mean surface areas (and SDs) for the left and right hemispheres, by handedness.

\begin{tabular}{lll}
\hline & Left-handers & Right-handers \\
\hline Left hemisphere surface area & $87855.1(7717.6)$ & 87984.5 (8469.9) \\
Right hemisphere surface area & $87817.2(8133.5)$ & $88295.6(8487.4)$
\end{tabular}

Table 2 | Repeated-measures ANOVA results from testing for an association between handedness and total hemispheric cortical surface areas.

\begin{tabular}{lccc}
\hline & \multicolumn{3}{c}{ Repeated-measures ANOVA } \\
\cline { 2 - 4 } & $\boldsymbol{P}$ & $\boldsymbol{F}$ & Partial $\eta^{\mathbf{2}}$ \\
\hline Handedness & 0.114 & 2.501 & 0.001 \\
Handedness * Hemisphere & 0.132 & 2.266 & 0.001 \\
Sex & $<0.001$ & 1193.7 & 0.367 \\
Age & $<0.001$ & 90.1 & 0.042 \\
Scanner field strength & $<0.001$ & 12.48 & 0.006 \\
\hline
\end{tabular}

Supplementary Material, together with descriptive statistics of all metrics, per handedness group.

\section{DISCUSSION}

In a large sample of primarily young adult and healthy individuals, we tested for associations of handedness with total and regional measures of hemispheric cerebral cortical surface area. We report on the largest sample to have been analyzed to date in relation to this question. The proportion of left-handers in our sample was lower than in the general population, due to an exclusion of left-handers from some of the smaller studies that were pooled to create our BIG dataset. This exclusion bias, however, did not affect the heterogeneity of scan parameters present in both handedness groups, as reflected in the similar usage of headcoils between them. Nonetheless, we observed a sex difference in the incidence of left-handedness that was consistent with previous literature (with left-handedness occurring at an elevated rate in males; Sommer et al., 2008).

We did not observe any difference in bilateral cortical surface area in left-handers compared to right-handers. Nor did we find significant evidence for associations of handedness with regionspecific bilateral surface areas, or their asymmetries, for regions related to language, hand motor control, or visual processing (Foundas et al., 1998, 1995; Willems et al., 2010). Our data therefore, provide little support for previously reported region-specific associations with handedness, although the Destrieux atlas' definitions of regions might not be identical to the definitions used in these previous studies. For example, the PT in the Destrieux atlas extends parietally (Destrieux et al., 2010), which is not a classic neuroanatomical definition (Geschwind and Levitsky, 1968; Steinmetz et al., 1991).

A limitation of our study was that, due to our large sample size and the number of cortical regions analyzed, systematic manual checking and adjustment of the automated parcellations was not feasible. Visual checks were made for only a small minority of images and not targeted to specific regions. However we exploited our subset of twice-scanned subjects in order to exclude regions that were not consistently parcellated from scan to re-scan, and also used outlier exclusion, as two forms of quality control. Clearly there is a need for improved methods of automated parcellation that capture some of the more variable and anatomically complex cortical regions better, in order to carry out future studies based on thousands of images. Another caveat is that the left and right definitions of cortical regions can only be considered "homologous" on the basis of information that was used in constructing the Destrieux atlas (that included information on cytoarchitecture), but this does not necessarily imply strict homology in genetic/developmental terms.

We found a suggestive association of handedness with the bilateral surface area of the superior part of the precentral sulcus, a region overlapping primary motor cortex. However, this association did not survive correction for multiple testing. Lefthanders showed reduced surface areas compared to right handers in our sample (Table 4), which is at least consistent with the findings reported by Amunts et al. (1996) and Foundas et al. (1998). Males tend to have larger brains than females, which was also the case in our dataset, but this observed trend of decreased cerebral cortical surface area in left-handers was independent of this sex effect, and in the opposite direction to what might be predicted by it. Another suggestive association was found bilaterally with the inferior temporal gyrus. Again, left-handers in our sample showed reduced surface areas bilaterally (Table 4).

Our broader screen of non-candidate regional surface area and asymmetry differences between left- and right-handers did not identify significant novel associations. While relatively large, our sample size allowed us to detect standardized effect sizes regarded as medium (http://imaging.mrc-cbu.cam.ac.uk/ statswiki/FAQ/effectSize), both before and after adjustment for multiple comparisons.

Although our dataset included a degree of heterogeneity in terms of scanning parameters used, there was no systematic difference in parameters applied for left- and right-handers, and we only analyzed measurements that showed a high scan-rescan correlation in twice-scanned subjects, despite this heterogeneity. Future studies based on even larger datasets will likely be affected by the same issue of heterogeneity, since large datasets are typically achieved through data pooling from multiple sources. It is therefore, encouraging that most of our measurements showed high scan-rescan correlations regardless of scanning heterogeneity.

An important issue in research on handedness is how exactly to define the trait. Many approaches have been taken to measure hand preference, ranging from motor performance measurements (e.g., relative hand skill, relative grip-strength; see Clerke and Clerke, 2001, for a brief overview); to self-report inventories assessing hand choice across various manual activities (Crovitz and Zener, 1962; Annett, 1967; Oldfield, 1971). Handedness inventories that account for preference across a range of tasks yield a rich assessment of (the degree of) handedness, and a detailed picture of its inter-subject variability. However, the resulting data are usually bimodal and are often subsequently dichotomized. For example, (Tan, 1993) showed 


\begin{tabular}{|c|c|c|c|c|}
\hline & & \multicolumn{3}{|c|}{ Repeated-measures ANOVA } \\
\hline \multirow[t]{2}{*}{ Opercular part of the interiorfrontal gyrus } & Handedness & 0.73 & 0.12 & $<0.001$ \\
\hline & Handedness $*$ Hemisphere & 0.63 & 0.23 & $<0.001$ \\
\hline Triangular part of the inferior frontal gyrus & Handedness & 0.88 & 0.02 & $<0.001$ \\
\hline \multirow[t]{2}{*}{ Anterior transverse temporal gyrus (of Heschl) } & Handedness & 0.86 & 0.03 & $<0.001$ \\
\hline & Handedness $*$ Hemisphere & 0.06 & 3.4 & 0.002 \\
\hline \multirow[t]{2}{*}{ Lateral aspect of the superior temporal gyrus } & Handedness & 0.57 & 0.33 & $<0.001$ \\
\hline & Handedness $*$ Hemisphere & 0.36 & 0.85 & $<0.001$ \\
\hline Planum temporale & Handedness & 0.42 & 0.64 & $<0.001$ \\
\hline & Handedness $*$ Hemisphere & 0.6 & 0.28 & $<0.001$ \\
\hline \multirow[t]{2}{*}{ Inferior part of the precentral sulcus } & Handedness & 0.76 & 0.09 & $<0.001$ \\
\hline & Handedness $*$ Hemisphere & 0.85 & 0.03 & $<0.001$ \\
\hline \multicolumn{5}{|l|}{ VISUAL-RELATED } \\
\hline \multirow[t]{2}{*}{ Inferior temporal gyrus } & Handedness & 0.037 & 4.36 & 0.002 \\
\hline & Handedness $*$ Hemisphere & 0.58 & 0.3 & $<0.001$ \\
\hline \multirow[t]{2}{*}{ Lateral occipito-temporal gyrus (fusiform gyrus) } & Handedness & 0.17 & 1.87 & 0.001 \\
\hline & Handedness $*$ Hemisphere & 0.53 & 0.4 & $<0.001$ \\
\hline \multirow[t]{2}{*}{ Lingual part of the medial occipito-temporal gyrus } & Handedness & 0.26 & 1.27 & 0.001 \\
\hline & Handedness $*$ Hemisphere & 0.1 & 2.67 & 0.001 \\
\hline
\end{tabular}

Reported are p-values before correction for multiple testing (none survived this correction).

Table 4 | Means (and SDs) for the superior part of the precentral sulcus, and inferior temporal gyrus, by hemisphere and handedness group.

\begin{tabular}{lcccc}
\hline & \multicolumn{2}{c}{ Left hemisphere } & & \multicolumn{1}{c}{ Right hemisphere } \\
\cline { 2 - 4 } & Left-handers & Right-handers & Left-handers & Right-handers \\
\hline $\begin{array}{l}\text { Superior part of the precentral sulcus } \\
\text { Inferior temporal gyrus }\end{array}$ & $914.9(207.5)$ & $952.7(200.9)$ & $965.1(201.9)$ & $990.4(214.8)$ \\
\hline
\end{tabular}

that hand preference, when assessed by a very detailed questionnaire (Waterloo handedness questionnaire; Steenhuis and Bryden, 1989), shows a clear distinction between left-handed and right-handed populations. Further evidence for an intrinsic dichotomy in handedness was also provided by McManus (1991) who observed the same proportion of left-handers regardless of the questionnaire used. Accordingly, simple self-assessments of overall handedness, such as that used in the present study (asking subjects only to categorize themselves as left- or right-handed) show close agreement with dichotomous scoring of handedness as derived from multi-item inventories, as well as robust testretest repeatability (Bryden et al., 1991; Tan, 1993; Ransil and Schachter, 1994). We are therefore confident of the validity of the binary, self-reported assessment of handedness that was used in our study.

Identifying cortical regional correlates of handedness may prove particularly useful in providing endophenotypes for future genetic studies of this trait, as well as clarifying the relationships between this and other forms of cerebral lateralization (Ocklenburg et al., 2013; Willems et al., 2014). We note that an association between handedness and cerebral cortical anatomy does not necessarily imply a simple causative relationship between the two. While it is conceivable that hand preference may arise due to hemispheric differences in cortical anatomy and function, it is equally conceivable that hand preference exerts developmental effects on cerebral cortical anatomy and function. 
Table 5 | Summary results for non-candidate cortical regions that achieved nominal significance in ANOVA.

\begin{tabular}{|c|c|c|c|c|}
\hline \multicolumn{2}{|l|}{ Cortical surface areas } & \multicolumn{3}{|c|}{ Repeated-measures ANOVA } \\
\hline & Handedness $*$ Hemisphere & 0.023 & 5.18 & 0.003 \\
\hline \multirow[t]{2}{*}{ Middle-anterior part of the cingulate gyrus and sulcus (aMCC) } & Handedness & 0.67 & 0.18 & $<0.001$ \\
\hline & Handedness $*$ Hemisphere & 0.003 & 8.99 & 0.005 \\
\hline Superior occipital gyrus (O1) & Handedness $*$ Hemisphere & 0.255 & 1.3 & $<0.001$ \\
\hline \multirow[t]{2}{*}{ Posterior transverse collateral sulcus } & Handedness & 0.648 & 0.21 & $<0.001$ \\
\hline & Handedness $*$ Hemisphere & 0.048 & 3.92 & 0.002 \\
\hline \multirow[t]{2}{*}{ Superior frontal sulcus } & Handedness & 0.038 & 4.31 & 0.002 \\
\hline & Handedness $*$ Hemisphere & 0.221 & 1.5 & $<0.001$ \\
\hline Parieto-occipital sulcus (or fissure) & Handedness $*$ Hemisphere & 0.25 & 1.32 & $<0.001$ \\
\hline
\end{tabular}

None of these results survived correction for multiple testing. Complete results for all regions and covariates are in Supplementary Table 1.

Table 6 | Means (and SDs) for non-candidate cortical regions that achieved nominal significance in ANOVA, by hemisphere and handedness group.

\begin{tabular}{lcccc}
\hline & \multicolumn{2}{c}{ Left hemisphere } & & \multicolumn{2}{c}{ Right hemisphere } \\
& Left-handers & Right-handers & Left-handers & Right-handers \\
\hline Anterior part of the cingulate gyrus and sulcus (ACC) & $1648.0(223.2)$ & $1707.4(264.5)$ & $1998.8(251.3)$ \\
Middle-anterior part of the cingulate gyrus and sulcus & $974.6(144.7)$ & $1014.5(170.3)$ & $1144.0(162.7)$ & $2016.7(271.1)$ \\
Superior occipital gyrus (O1) & $1131.5(166.2)$ & $1101.1(167.7)$ & $1251.1(177.8)$ & $1114.1(169.8)$ \\
Posterior transverse collateral sulcus & $300.7(70.9)$ & $294.2(66.4)$ & $373.0(98.8)$ & $386.9(98.8)$ \\
Superior frontal sulcus & $2004.9(286.8)$ & $2077.1(302.7)$ & $1867.6(271.7)$ & $1906.7(296.1)$ \\
Sulcus intermedius primus (of Jensen) & $280.2(145.6)$ & $257.6(127.3)$ & $350.1(150.3)$ & $364.0(151.7)$ \\
Parieto-occipital sulcus (or fissure) & $1445.9(225.9)$ & $1429.0(239.3)$ & $1584.5(265.8)$ & $1544.2(255.8)$ \\
\hline
\end{tabular}

As noted in the Introduction, there is strong evidence indicating that motor asymmetry of the arms and hands is initiated very early during human embryonic development, possibly even before the cerebral cortex exerts significant influence (Hepper, 2013). These early motor asymmetries, potentially under spinomuscular control, could therefore contribute to the determination of both handedness and regional cortical development.

Left-handed people show increased rates of reductions or reversals of lateralized brain functions, compared to righthanders (reviewed by Willems et al., 2014). Functional imaging studies of left-handers allow the possibility to study not only basic lateralization of brain function (e.g., of face perception), but also embodied cognition, and the extent of co-lateralization of different cognitive functions (Willems et al., 2014). Our survey of cerebral anatomical correlates of handedness may serve to inform these investigations, as it can suggest a prioritization of specific regions and cognitive processes to focus on with functional imaging techniques.
It is clear from our results, and those of previous studies, that any changes in brain structure associated with left-handedness are subtle. As noted earlier, it is likely that the genetic contributions to left-handedness are heterogeneous in nature, with multiple different genes being involved, and the same may be true of environmental influences (which also remain poorly understood). Etiologic heterogeneity suggests that there will be different forms of left-handedness which may manifest differently in terms of how striking any brain structural and functional correlates may be, and also differently in how, and to what extent, other lateralized cognitive systems are re-organized. A promising approach for studying the relations between lateralization and cognition will therefore be to specifically recruit left-handers, in order to recruit sufficient numbers for characterizing their heterogeneity, followed by assessments of brain structure and function in addition to neuropsychological testing, and genetic analysis (Marie et al., 2013; Mellet et al., 2013). 


\section{ACKNOWLEDGMENTS}

We wish to thank Han Brunner for his involvement in the creation and growth of the BIG (Brain Imaging Genetics) dataset and to all persons who kindly participated in the BIG research. This work makes use of the BIG (Brain Imaging Genetics) database, first established in Nijmegen, The Netherlands, in 2007. This resource is now part of Cognomics (www.cognomics. $\mathrm{nl}$ ), a joint initiative by researchers of the Donders Centre for Cognitive Neuroimaging, the Human Genetics and Cognitive Neuroscience departments of the Radboud university medical centre and the Max Planck Institute for Psycholinguistics in Nijmegen. The Cognomics Initiative is supported by the participating departments and centres and by external grants, i.e., the Biobanking and Biomolecular Resources Research Infrastructure (Netherlands) (BBMRI-NL), the Hersenstichting Nederland, and the Netherlands Organization for Scientific Research (NWO).

\section{SUPPLEMENTARY MATERIAL}

The Supplementary Material for this article can be found online at: http://www.frontiersin.org/journal/10.3389/fpsyg. 2014.00261/abstract

\section{REFERENCES}

Amunts, K., Schlaug, G., Schleicher, A., Steinmetz, H., Dabringhaus, A., Roland, P. E., et al. (1996). Asymmetry in the human motor cortex and handedness. Neuroimage 4, 216-222. doi: 10.1006/nimg.1996.0073

Annett, M. (1967). The binomial distribution of right, mixed and left handedness. Q. J. Exp. Psychol. 19, 327-33. doi: 10.1080/14640746708400109

Annett, M. (1985). Left, Right, Hand and Brain: The Right Shift Theory. London: Laurence Erlbaum Associates.

Armour, J. A., Davison, A., and McManus, I. C. (2014). Genome-wide association study of handedness excludes simple genetic models. Heredity 112, 221-225. doi: 10.1038/hdy.2013.93

Arning, L., Ocklenburg, S., Schulz, S., Ness, V., Gerding, W. M., Hengstler, J. G., et al. (2013). VNTR polymorphism is associated with degree of handedness but not direction of handedness. PLoS ONE 8:e67251. doi: 10.1371/journal.pone.0067251

Badzakova-Trajkov, G., Häberling, I. S., Roberts, R. P., and Corballis, M. C. (2010). Cerebral asymmetries: complementary and independent processes. PLoS ONE 5:e9682. doi: 10.1371/journal.pone.0009682

Bloss, C. S., Delis, D. C., Salmon, D. P., and Bondi, M. W. (2010). APOE genotype is associated with left-handedness and visuospatial skills in children. Neurobiol. Aging 31, 787-795. doi: 10.1016/j.neurobiolaging.2008.05.021

Brandler, W. M., Morris, A. P., Evans, D. M., Scerri, T. S., Kemp, J. P., Timpson, N. J., et al. (2013). Common variants in left/right asymmetry genes and pathways are associated with relative hand skill. PLoS Genet. 9:e1003751. doi: 10.1371/journal.pgen.1003751

Bryden, M. P., McManus, I. C., and Steenhuis, R. E. (1991). Handedness is not related to self-reported disease incidence. Cortex 27, 605-611. doi: 10.1016/S0010-9452(13)80009-3

Bukowski, H., Dricot, L., Hanseeuw, B., and Rossion, B. (2013). Cerebral lateralization of face-sensitive areas in left-handers: only the FFA does not get it right. Cortex 49, 2583-2589. doi: 10.1016/j.cortex.2013.05.002

Clerke, A., and Clerke, J. (2001). A literature review of the effect of handedness on isometric grip strength differences of the left and right hands. Am. J. Occup. Ther. 55, 206-211. doi: 10.5014/ajot.55.2.206

Corballis, M. C., Badzakova-Trajkov, G., and Häberling, I. S. (2012). Right hand, left brain: genetic and evolutionary bases of cerebral asymmetries for language and manual action. Wiley Interdiscip. Rev. Cogn. Sci. 3, 1-17. doi: $10.1002 /$ wcs. 158

Coren, S., and Porac, C. (1977). Fifty centuries of right-handedness: the historical record. Science 198, 631-632. doi: 10.1126/science.335510

Crovitz, H. F., and Zener, K. (1962). A group-test for assessing hand- and eyedominance. Am. J. Psychol. 75, 271-276. doi: 10.2307/1419611 de Leon, M. J., la Regina, M. E., Ferris, S. H., Gentes, C. I., and Miller, J. D. (1986). Reduced incidence of left-handedness in clinically diagnosed dementia of the Alzheimer type. Neurobiol. Aging 7, 161-164. doi: 10.1016/01974580(86)90037-0

Destrieux, C., Fischl, B., Dale, A., and Halgren, E. (2010). Automatic parcellation of human cortical gyri and sulci using standard anatomical nomenclature. Neuroimage 53, 1-15. doi: 10.1016/j.neuroimage.2010.06.010

Faul, F., Erdfelder, E., Buchner, A., and Lang, A.-G. (2009). Statistical power analyses using $\mathrm{G}^{*}$ Power 3.1: tests for correlation and regression analyses. Behav. Res. Methods 41, 1149-1160. doi: 10.3758/BRM.41.4.1149

Faurie, C., and Raymond, M. (2004). Handedness frequency over more than ten thousand years. Proc. Biol. Sci. 7, S43-S45. doi: 10.1098/rsbl.2003.0092

Fischl, B., van der Kouwe, A., Destrieux, C., Halgren, E., Segonne, F., Salat, D. H., et al. (2004). Automatically parcellating the human cerebral cortex. Cereb. Cortex 14, 11-22. doi: 10.1093/cercor/bhg087

Foundas, A. L., Hong, K., Leonard, C. M., and Heilman, K. M. (1998). Hand preference and magnetic resonance imaging asymmetries of the central sulcus. Neuropsychiatry Neuropsychol. Behav. Neurol. 11, 65-71.

Foundas, A. L., Leonard, C. M., Heilman, K. M. (1995). Morphologic cerebral asymmetries and handedness. The pars triangularis and planum temporale. Arch. Neurol. 52, 501-508. doi: 10.1001/archneur.1995.00540290091023

Francks, C., Maegawa, S., Lauren, J., Abrahams, B., Velayos-Baeza, A., Medland, S., et al. (2007). LRRTM1 on chromosome 2 p12 is a maternally suppressed gene that is associated paternally with handedness and schizophrenia. Mol. Psychiatry 12, 1129-1139. doi: 10.1038/sj.mp.4002053

Franke, B., Vasquez, A. A., Veltman, J. A., Brunner, H. G., Rijpkema, M., and Fernandez, G. (2010). Genetic variation in CACNA1C, a gene associated with bipolar disorder, influences brainstem rather than gray matter volume in healthy individuals. Biol. Psychiatry 68, 586-588. doi: 10.1016/j.biopsych.2010. 05.037

Geschwind, N., and Behan, P. (1982). Left-handedness: association with immune disease, migraine, and developmental learning disorder. Proc. Natl. Acad. Sci. U.S.A. 79, 5097-5100. doi: 10.1073/pnas.79.16.5097

Geschwind, N., and Levitsky, W. (1968). Human brain: left-right asymmetries in temporal speech region. Science 161, 186-187. doi: 10.1126/science.161.3837.186

Good, C. D., Johnsrude, I., Ashburner, J., Henson, R. N. A., Friston, K. J., Frackowiak, R. S. (2001). Cerebral asymmetry and the effects of sex and handedness on brain structure: a voxel-based morphometric analysis of 465 normal adult human brains. Neuroimage 14, 685-700. doi: 10.1006/nimg.2001.0857

Gordon, H. W., and Kravetz, S. (1991). The influence of gender, handedness, and performance level on specialized cognitive functioning. Brain Cogn. 15, 37-61. doi: 10.1016/0278-2626(91)90014-Y

Halpern, D. F., Haviland, M. G., and Killian, C. D. (1998). Handedness and sex differences in intelligence: evidence from the medical college admission test. Brain Cogn. 38, 87-101. doi: 10.1006/brcg.1998.1021

Hardyck, C., and Petrinovich, L. F. (1977). Left-handedness. Psychol. Bull. 84, 385-404. doi: 10.1037/0033-2909.84.3.385

Hepper, P. G. (2013). The developmental origins of laterality: fetal handedness. Dev. Psychobiol. 55, 588-595. doi: 10.1002/dev.21119

Herve, P. V., Mazoyer, B., Crivello, F., Perchey, G., and Tzourio-Mazoyer, N. (2005). Finger tapping, handedness and grey matter amount in the Rolando's genu area. Neuroimage 25, 1133-1145. doi: 10.1016/j.neuroimage.2004. 12.062

Herve, P. Y., Crivello, F., Perchey, G., Mazoyer, B., and Tzourio-Mazoyer, N. (2006). Handedness and cerebral anatomical asymmetries in young adult males. Neuroimage 29, 1066-1079. doi: 10.1016/j.neuroimage.2005.08.031

Kloppel, S., van Eimeren, T., Glauche, V., Vongerichten, A., Munchau, A., Frackowiak, R. S., et al. (2007). The effect of handedness on cortical motor activation during simple bilateral movements. Neuroimage 34, 274-280. doi: 10.1016/j.neuroimage.2006.08.038

Knecht, S., Deppe, M., Drager, B., Bobe, L., Lohmann, H., Ringelstein, E. B., et al. (2000a). Language lateralization in healthy right-handers. Brain 123, 74-81. doi: 10.1093/brain/123.1.74

Knecht, S., Drager, B., Deppe, M., Bobe, L., Lohmann, H., Floel, A., et al. (2000b) Handedness and hemispheric language dominance in healthy humans. Brain 123, 2512-2518. doi: 10.1093/brain/123.12.2512

London, W. P. (1989). Left-handedness and life expectancy. Percept. Mot. Skills 68(3 pt 2), 1040-1042. doi: 10.2466/pms.1989.68.3c.1040 
Lonsdorf, E. V., and Hopkins, W. D. (2005). Wild chimpanzees show populationlevel handedness for tool use. Proc. Natl. Acad. Sci. U.S.A. 102, 12634-12638. doi: $10.1073 /$ pnas.0505806102

Luh, K. E., Redl, J., and Levy, J. (1994). Left- and right-handers see people differently: free-vision perceptual asymmetries for chimeric stimuli. Brain Cogn. 25, 141-160. doi: 10.1006/brcg.1994.1028

Marie, D., Jobard, G., Crivello, F., Perchey, G., Petit, L., Mellet, E., et al. (2013). Descriptive anatomy of Heschl's gyri in 430 healthy volunteers, including 198 left-handers. Brain Struct. Funct. 6:6. doi: 10.1007/s00429-0130680-x

McManus, I. C. (1985). Right- and left-hand skill: failure of the right shift model. Br. J. Psychol. 76(pt 1), 1-34. doi: 10.1111/j.2044-8295.1985.tb 01926.x

McManus, I. C. (1991). The inheritance of left-handedness. Ciba Found. Symp. 162, 251-267.

McManus, I. C.( 2009). "The history and geography of human handedness," in Language Lateralisation and Psychosis, eds E. C. Sommer and R. Kahn (Cambridge: Cambridge University Press), 37-57.

McManus, I. C., Davison, A., and Armour, J. A. (2013). Multilocus genetic models of handedness closely resemble single-locus models in explaining family data and are compatible with genome-wide association studies. Ann. N.Y. Acad. Sci. 1288, 48-58. doi: 10.1111/nyas.12102

Medland, S. E. (2009). "Meta-analysis of GWAS for handedness: results from the ENGAGE consortium," in American Society of Human Genetics, Meeting Abstract. Available online at: http://www.ashg.org/2009meeting/abstracts/ fulltext/f21141.htm

Medland, S. E., Duffy, D. L., Spurdle, A. B., Wright, M. J., Geffen, G. M., Montgomery, G. W., et al. (2005). Opposite effects of androgen receptor CAG repeat length on increased risk of left-handedness in males and females. Behav. Genet. 35, 735-744. doi: 10.1007/s10519-005-6187-3

Medland, S. E., Duffy, D. L., Wright, M. J., Geffen, G. M., Hay, D. A., Levy, F., et al. (2009). Genetic influences on handedness: data from 25,732 Australian and Dutch twin families. Neuropsychologia 47, 330-337. doi: 10.1016/j.neuropsychologia.2008.09.005

Mellet, E., Jobard, G., Zago, L., Crivello, F., Petit, L., Joliot, M., et al. (2013). Relationships between hand laterality and verbal and spatial skills in 436 healthy adults balanced for handedness. Laterality 19, 383. doi: 10.1080/1357650X.2013.796965

Meunier, H., Fizet, J., and Vauclair, J. (2013). Tonkean macaques communicate with their right hand. Brain Lang. 126, 181-187. doi: 10.1016/j.bandl.2013.05.004

Narr, K. L., Bilder, R. M., Luders, E., Thompson, P. M., Woods, R. P., Robinson, D., et al. (2007). Asymmetries of cortical shape: effects of handedness, sex and schizophrenia. Neuroimage 34, 939-948. doi: 10.1016/j.neuroimage.2006. 08.052

Ocklenburg, S., Beste, C., and Gunturkun, O. (2013). Handedness: a neurogenetic shift of perspective. Neurosci. Biobehav. Rev. 37(10 Pt 2), 2788-2793. doi 10.1016/j.neuroimage.2011.01.035

Oldfield, R. C. (1971). The assessment and analysis of handedness: the Edinburgh inventory. Neuropsychologia 9, 97-113. doi: 10.1016/0028-3932(71) 90067-4

Panizzon, M. S., Fennema-Notestine, C., Eyler, L. T., Jernigan, T. L., PromWormley, E., Neale, M., et al. (2009). Distinct genetic influences on cortical surface area and cortical thickness. Cereb. Cortex 19, 2728-2735. doi: 10.1093/cercor/bhp026

Peters, M., Reimers, S., and Manning, J. T. (2006). Hand preference for writing and associations with selected demographic and behavioral variables in 255,100 subjects: the BBC internet study. Brain Cogn. 62, 177-189. doi: 10.1016/j.bandc.2006.04.005

Powell, J. L., Kemp, G. J., Roberts, N., and Garcia-Finana, M. (2012). Sulcal morphology and volume of Broca's area linked to handedness and sex. Brain Lang. 121, 206-218. doi: 10.1016/j.bandl.2012.03.003
Ransil, B. J., and Schachter, S. C. (1994). Test-retest reliability of the Edinburgh handedness inventory and global handedness preference measurements, and their correlation. Percept. Mot. Skills 79(3 pt 1), 1355-1372. doi: 10.2466/pms.1994.79.3.1355

Savitz, J., van der Merwe, L., Solms, M., and Ramesar, R. (2007). Lateralization of hand skill in bipolar affective disorder. Genes Brain Behav. 6, 698-705. doi: 10.1111/j.1601-183X.2006.00299.x

Scerri, T. S., Brandler, W. M., Paracchini, S., Morris, A. P., Ring, S. M., Richardson, A. J., et al. (2011). PCSK6 is associated with handedness in individuals with dyslexia. Hum. Mol. Genet. 20, 608-614. doi: 10.1093/hmg/ddq475

Sommer, I. E., Aleman, A., Somers, M., Boks, M. P., and Kahn, R. S. (2008). Sex differences in handedness, asymmetry of the Planum Temporale and functional language lateralization. Brain Res. 1206, 76-88. doi: 10.1016/j.brainres.2008.01.003

Steenhuis, R. E., and Bryden, M. P. (1989). Different dimensions of hand preference that relate to skilled and unskilled activities. Cortex 25, 289-304. doi: 10.1016/S0010-9452(89)80044-9

Steinmetz, H., Volkmann, J., Jancke, L., and Freund, H. J. (1991). Anatomical leftright asymmetry of language-related temporal cortex is different in left- and right-handers. Ann. Neurol. 29, 315-319. doi: 10.1002/ana.410290314

Tan, U. (1993). Normal distribution of hand preference and its bimodality. Int. J. Neurosci. 68, 61-65. doi: 10.3109/00207459308994260

Tzourio, N., Crivello, F., Mellet, E., Nkanga-Ngila, B., and Mazoyer, B. (1998). Functional anatomy of dominance for speech comprehension in left handers vs right handers. Neuroimage 8, 1-16. doi: 10.1006/nimg.1998.0343

Vallortigara, G., Chiandetti, C., and Sovrano, V. A. (2011). Brain asymmetry (animal). Wiley Interdiscip. Rev. Cogn. Sci. 2, 146-157. doi: 10.1002/wcs.100

Vuoksimaa, E., Koskenvuo, M., Rose, R. J., and Kaprio, J. (2009). Origins of handedness: a nationwide study of 30,161 adults. Neuropsychologia 47, 1294-1301. doi: 10.1016/j.neuropsychologia.2009.01.007

Willems, R. M., der Haegen, L. V., Fisher, S. E., and Francks, C. (2014). On the other hand: including left-handers in cognitive neuroscience and neurogenetics. Nat. Rev. Neurosci. 15, 193-201. doi: 10.1038/nrn3679

Willems, R. M., and Hagoort, P. (2009). Hand preference influences neural correlates of action observation. Brain Res. 7, 90-104. doi: 10.1016/j.brainres.2009.02.057

Willems, R. M., Peelen, M. V., and Hagoort, P. (2010). Cerebral lateralization of face-selective and body-selective visual areas depends on handedness. Cereb. Cortex 20, 1719-1725. doi: 10.1093/cercor/bhp234

Witelson, S. F., and Kigar, D. L. (1992). Sylvian fissure morphology and asymmetry in men and women: bilateral differences in relation to handedness in men. J. Comp. Neurol. 323, 326-340. doi: 10.1002/cne.903230303

Conflict of Interest Statement: The authors declare that the research was conducted in the absence of any commercial or financial relationships that could be construed as a potential conflict of interest.

Received: 10 January 2014; accepted: 11 March 2014; published online: 28 March 2014 Citation: Guadalupe T, Willems RM, Zwiers MP, Arias Vasquez A, Hoogman M, Hagoort P, Fernandez G, Buitelaar J, Franke B, Fisher SE and Francks C (2014) Differences in cerebral cortical anatomy of left- and right-handers. Front. Psychol. 5:261. doi: 10.3389/fpsyg.2014.00261

This article was submitted to Cognition, a section of the journal Frontiers in Psychology.

Copyright (C) 2014 Guadalupe, Willems, Zwiers, Arias Vasquez, Hoogman, Hagoort, Fernandez, Buitelaar, Franke, Fisher and Francks. This is an open-access article distributed under the terms of the Creative Commons Attribution License (CC BY). The use, distribution or reproduction in other forums is permitted, provided the original author(s) or licensor are credited and that the original publication in this journal is cited, in accordance with accepted academic practice. No use, distribution or reproduction is permitted which does not comply with these terms. 